ISSN 2693-2326

\title{
Outcome of HIV Partner Notification Service Among People Living With HIV Infection in North-Central Nigeria
}

\author{
International Journal of Infectious Diseases and Research
}

Research Article

Abayomi Joseph Afe ${ }^{1 *}$, Sekinat owoyemi ${ }^{1}$, Hope Emmanuel ${ }^{1}$, Onyema Maduakalom ${ }^{1}$, Ganiyu Agboola ${ }^{1}$, Timothy Akinmurele $^{1}$

\author{
*Correspondence author \\ Dr. Abayomi Joseph Afe; MBBS, MPH, Ph.D., \\ FRSPH \\ Community Medicine Department \\ Equitable Health Access Initiative \\ Lagos \\ Nigeria.
}

${ }^{1}$ Equitable Health Access Initiative, Lagos, Nigeria

Submitted : 13 Jan 2021 ; Published : 31 Jan 2021

\begin{abstract}
Introduction: According to the Nigeria National HIV/AIDS Indicator and Impact Survey (NAIIS) conducted in 2018 but released in 2019 the current HIV prevalence in Nigeria is 1.4\% among adults aged 15-49 years. This implies that there are about 1.9 million people living with HIV in Nigeria. Yet the percentage of Nigerians that had access to HIV counselling and testing services was just $37 \%$ in 2018 and about $46.9 \%$ of PLHIV aged 15-64 years knew their HIV status according to NAIIS 2018 report. . To improve on the HIV testing coverage, HIV testing services programming must be focused on utilizing strategies that maximise efficiency while expanding access to accurate, high-quality services. One of such strategies is to prioritise index case testing services for sexual partners of index clients. Partner Notification Services (PNS) Partner HIV Testing service is a type of index case testing among sexual network of an index case.
\end{abstract}

Methods: Partner Notification Services (PNS) is a voluntary process where sexual partners of HIV-infected persons are contacted, counselled and are offered HIV test administered by a trained counselor. Each infected contact can then become the starting point for new contact tracing, until no more contacts can be found. This study was a retrospective review of the hospital records of HIV positive clients who participated in the HIV partner notification services.

Results: A total of 705 HIV patients (Female-68\%, Male-32\%) enrolled in care at the two centers accessed the HIV partner notification services (PNS). These index cases were counselled and brought their 719 sexual partners to the hospitals for HIV testing. About $27 \%$ of the sexual partners were HIV infected and $71 \%$ were HIV negative while $2 \%$ were of unknown HIV status because they did not take the HIV test. Viral load suppression rate was about $48 \%$ among the index cases., more female are virally suppressed than men (71\% vs 29\%). About $100 \%$ of the index with suppressed viral load had one sexual partner while for index cases with unsuppressed viral load $80 \%$ had one sexual partner, 19\% had two sexual partners and 1\% had 3 sexual partners. The frequency of unmarried sexual partners was higher among index cases with suppressed viral load (22\%) compared with index cases with unsuppressed viral load (36\%). Surprisingly, incidence of HIV infection was higher at 36\% among partners of index cases with suppressed viral load when compared with HIV incidence of 11\% among partners of index with unsuppressed viral load.

Conclusion and Recommendations: Uptake of PNS was 100\% among index cases while 98\% of their sexual contacts received HIV testing services. This shows PNS as a very effective strategy in increasing HIV testing services. Likewise, the HIV testing yield of $27 \%$ was higher than the yield from routine walk-in clients at the centers. It is therefore recommended that PNS be the standard of care at all centers offering HIV testing services.

\section{Introduction}

\section{Background}

Globally, there are 38 million people living with HIV (PLHIV) of which 25.4 million people are on treatment in 2020. This implies there are 12.6 million people still awaiting antiretroviral treatment.
However, new HIV infections have been reduced by $23 \%$ since 2010 , due largely to a substantial decrease of $38 \%$ in eastern and southern Africa. But HIV infections have increased by $72 \%$ in Eastern Europe and central Asia, by $22 \%$ in the Middle East and North Africa and by $21 \%$ in Latin America. Worldwide, there were still 690000 AIDS-related deaths in 2019 and 1.7 million 
new infections. The 2020 targets of reducing AIDS-related deaths to fewer than 500000 and new HIV infections to fewer than 500 000 can no longer be achieved due partly to COVID-19 pandemic; even though the UNAIDS 90:90:90 was off course before the onset of the coronavirus infection [1].

According to the Nigeria National HIV/AIDS Indicator and Impact Survey (NAIIS) conducted in 2018 but released in 2019 the current HIV prevalence in Nigeria is $1.4 \%$ among adults aged 15-49 years. This implies that there are about 1.9 million people living with HIV in Nigeria. However, women aged 15-49 years are more than twice as likely to be living with HIV than men $(1.9 \%$ versus $0.9 \%)$ The difference in HIV prevalence between women and men is greatest among younger adults, with young women aged 20-24 years more than three times as likely to be living with HIV as young men in the same age group. Among children aged $0-14$ years, HIV prevalence is $0.2 \%$. The SouthSouth zone of the country has the highest HIV prevalence, at 3.1\% among adults aged 15-49 years. HIV prevalence is also high in the North Central zone (2.0\%) and in the South East zone (1.9\%). HIV prevalence is lower in the South West zone (1.1\%), the North East zone (1.1\%) and the North West zone (0.6\%) [2].In subSaharan Africa, young women and adolescent girls accounted for one in four new infections in 2019, despite making up about $10 \%$ of the total population. This has been attributed to gender-based violence and gender inequalities in healthcare access.

Nigeria adopted the test and treat policy in 2016 which translates to initiating antiretroviral treatment to all HIV infected persons. This measure has led to almost tripled the number of people living with HIV who are on antiretroviral therapy from 360000 people in 2010 to more than 1 million people in 2018 [3]. Excellent adherence to antiretroviral therapy leads to viral suppression, which promote good health in those living with HIV and prevent transmission of the virus. However, the NAIIS result indicate that more than half of people living with HIV still do not have suppressed viral loads.

Recent years, there has been a significant expansion in the country's response to HIV. The number of sites providing treatment has more than tripled, the number of sites providing services to prevent mother-to-child transmission of HIV has increased eightfold and the number of HIV counselling and testing sites has increased fourfold. A total of 11.3 million adults were counselled and tested for HIV in 2016, four times as many as in 2012. Yet the percentage of Nigerians that had access to HIV counselling and testing services was just $37 \%$ in 2018 . While only about $46.9 \%$ of PLHIV aged 15-64 years knew their HIV status according to NAIIS 2018 report. To improve on the HIV testing coverage, HIV testing services programming must be focused on utilizing strategies that maximise efficiency while expanding access to accurate, high-quality services. One of such strategies is to prioritise index case testing services for children of positive women and partners of index clients in all settings especially in health facilities. A type of index case testing among sexual network is the partner HIV testing services or partner notification services (PNS).

HIV partner notification is also a form of contact tracing, a known strategy for controlling the spread of sexually transmitted infections (STIs). HIV partner notification service entails the engagement of the assistance of an HIV-positive individual for contact tracing to allow mapping of as many sexual contacts as possible, and encouraging these contacts to participate in HIV testing and treatment [4-7]. Each infected contact can then become the starting point for new contact tracing, until no more contacts can be found. Such a behavioural network strategy has been used successfully in some countries to identify undiagnosed cases of syphilis and HIV [4,5,6,8,9].

\section{Objectives}

The purpose of this paper was to review the outcome of the partner HIV testing services implemented in three public secondary health facilities in the northcentral region of Nigeria.

\section{Methods}

\section{Study Type: Retrospective study}

Study Design: Cross Sectional

Study Location: Antiretroviral clinics in three public secondary health facilities in Northcentral Nigeria.

Study Period: June 2018-June 2019

Protocol: Review of hospital records of HIV positive clients who participated in the HIV partner notification services

Inclusion Criteria

1. HIV positive clients

2. Enrolled in the ART programme of the hospital

3. Adults male and female

4. Must have participated in the Partner HIV testing services

\section{HIV counseling and testing}

Sexual partners of HIV-infected persons who were contacted and willing to take the HIV received a face-to-face pre-test counseling administered by a trained counselor. Usually the pre-test counseling lasted for about 30 to 45 minutes. A written consent for HIV testing was obtained from the client, after which venous blood was then collected by an experienced laboratory staff using sterilized needles and tubes. Plasma samples were coded by unique identification numbers and were screened for HIV antibody using a rapid testing antibody test kits, according to the Nigerian national testing algorithm. A serial HIV testing algorithm which specify the use of a highly sensitive RDT for test 1 . If test 1 is negative, then an HIV negative result is given to the client but if test 1 is HIV positive a further confirmation with a more specific RDT is used to test same sample. If test 2 is positive, then an HIV positive result is delivered to the client. All participants, no matter what the HIV testing results were, received routine post-test counseling and referral for other appropriate health services.

\section{Partner Notification Services}

Partner Notification Services (PNS) is also known as Index Case Testing, Index Client Testing or Contact Tracing. It is a voluntary process where trained health workers ask people living with HIV (PLHIV) about their sexual partners, trace the partners and offer HIV testing to the partners. This process can be initiated in HIV testing settings for newly diagnosed clients or in HIV treatment settings for clients already on antiretroviral treatment. Then with 
the consent of the HIV positive index clients, providers notify the sexual partners of their potential HIV exposure and offer HIV testing. Results

\begin{tabular}{|l|l|l|l|l|}
\hline Characteristics & Index case & Sexual Partners \\
\hline Gender & No & Frequency & No & Frequency \\
\hline Male & 228 & $32 \%$ & 484 & $67 \%$ \\
\hline Female & 477 & $68 \%$ & 235 & $33 \%$ \\
\hline Total & 705 & $100 \%$ & 719 & $100 \%$ \\
\hline Marital status & \multicolumn{5}{|l|}{} \\
\hline Unmarried & 204 & $29 \%$ & 210 & $29 \%$ \\
\hline Married partner & 501 & $71 \%$ & 509 & $71 \%$ \\
\hline Total & 705 & $100 \%$ & 719 & $100 \%$ \\
\hline HIV Status & No & Freq & No & Freq \\
\hline HIV Positive & 705 & $100 \%$ & 191 & $27 \%$ \\
\hline HIV Negative & 0 & 0 & 507 & $71 \%$ \\
\hline Unknown & 0 & 0 & 21 & $2 \%$ \\
\hline Total & 705 & $100 \%$ & 719 & $100 \%$ \\
\hline Viral load & \multicolumn{5}{|l|}{} \\
\hline Suppressed & 246 & $48 \%$ & & \\
\hline Unsuppressed & 270 & $52 \%$ & & \\
\hline Total & 516 & $100 \%$ & & \\
\hline
\end{tabular}

Table 1: Characteristics of Index Cases and their Sexual Partners

\begin{tabular}{|l|l|l|l|l|l|l|}
\hline \multicolumn{2}{|l}{ Demography Sexual Partners } & \multicolumn{2}{l|}{$\begin{array}{l}\text { HIV Positive (Concordant) } \\
\text { Partners }\end{array}$} & \multicolumn{2}{l|}{$\begin{array}{l}\text { HIV Negative Partners } \\
\text { (Discordant) }\end{array}$} \\
\hline Gender & Freq & Percent & Freq & Percent & Freq & Percent \\
\hline Male & 484 & $67 \%$ & 117 & $61 \%$ & 352 & $69 \%$ \\
\hline Female & 235 & $33 \%$ & 74 & $39 \%$ & 155 & $31 \%$ \\
\hline Total & $\mathbf{7 1 9}$ & $\mathbf{1 0 0} \%$ & $\mathbf{1 9 1}$ & $\mathbf{1 0 0} \%$ & $\mathbf{5 0 7}$ & $\mathbf{1 0 0 \%}$ \\
\hline Age & Freq & Percent & Freq & Percent & Freq & Percent \\
\hline $13-17$ & 3 & $\mathbf{0 \%}$ & 1 & $\mathbf{1 \%}$ & 3 & $\mathbf{1 \%}$ \\
\hline $18-22$ & 19 & $3 \%$ & 5 & $3 \%$ & 12 & $\mathbf{2 \%}$ \\
\hline $23-27$ & 65 & $9 \%$ & 26 & $\mathbf{1 4 \%}$ & 37 & $\mathbf{7 \%}$ \\
\hline $28-32$ & 150 & $21 \%$ & 40 & $21 \%$ & 110 & $22 \%$ \\
\hline $33-37$ & 107 & $15 \%$ & 27 & $14 \%$ & 76 & $15 \%$ \\
\hline $38-42$ & 184 & $26 \%$ & 53 & $28 \%$ & 124 & $24 \%$ \\
\hline $43-47$ & 84 & $12 \%$ & 19 & $10 \%$ & 62 & $12 \%$ \\
\hline $48-52$ & 54 & $8 \%$ & 10 & $5 \%$ & 43 & $8 \%$ \\
\hline $53-57$ & 30 & $4 \%$ & 4 & $2 \%$ & 25 & $5 \%$ \\
\hline $58-62$ & 14 & $2 \%$ & 4 & $2 \%$ & 9 & $2 \%$ \\
\hline $63-67$ & 2 & $0 \%$ & 0 & $0 \%$ & 2 & $0 \%$ \\
\hline $68-72$ & 5 & $1 \%$ & 2 & $1 \%$ & 2 & $0 \%$ \\
\hline $73-77$ & 1 & $0 \%$ & 0 & $0 \%$ & 1 & $0 \%$ \\
\hline $78-82$ & 1 & $0 \%$ & 0 & $0 \%$ & 0 & $0 \%$ \\
\hline $83-87$ & 0 & $0 \%$ & 0 & $0 \%$ & 1 & $0 \%$ \\
\hline Total & $\mathbf{7 1 9}$ & $\mathbf{1 0 0 \%}$ & $\mathbf{1 9 1}$ & $\mathbf{1 0 0 \%}$ & $\mathbf{5 0 7}$ & $\mathbf{1 0 0 \%}$ \\
\hline & & & & & \\
\hline
\end{tabular}




\begin{tabular}{|l|l|l|l|l|l|l|}
\hline Marital Status & Freq & Percent & Freq & Percent & Freq & Percent \\
\hline Married partner & 509 & $71 \%$ & $\mathbf{1 4 9}$ & $\mathbf{7 8 \%}$ & $\mathbf{3 4 3}$ & $\mathbf{6 8 \%}$ \\
\hline unmarried & 210 & $29 \%$ & 42 & $22 \%$ & 164 & $32 \%$ \\
\hline Total & 719 & $\mathbf{1 0 0} \%$ & 191 & $\mathbf{1 0 0 \%}$ & 507 & $\mathbf{1 0 0 \%}$ \\
\hline
\end{tabular}

Table2: HIV Test Results of Sexual Partners and their demography

\begin{tabular}{|c|c|c|c|c|}
\hline \multirow{2}{*}{$\begin{array}{l}\text { Characteristics } \\
\text { Gender } \\
\end{array}$} & \multirow{2}{*}{$\begin{array}{l}\text { Index case with } \\
\text { Suppressed VL } \\
\text { Frequency } \\
\end{array}$} & \multicolumn{3}{|c|}{ Index case with unsuppressed VL } \\
\hline & & Percent & Frequency & Percent \\
\hline Male & 72 & $29 \%$ & 96 & $36 \%$ \\
\hline Female & 174 & $71 \%$ & 173 & $64 \%$ \\
\hline Total & 246 & $100 \%$ & 269 & $100 \%$ \\
\hline \multicolumn{5}{|l|}{ Age } \\
\hline $13-17$ & 5 & $2 \%$ & 1 & $0 \%$ \\
\hline $18-22$ & 14 & $6 \%$ & 9 & $3 \%$ \\
\hline $23-27$ & 38 & $15 \%$ & 26 & $10 \%$ \\
\hline $28-32$ & 68 & $28 \%$ & 54 & $20 \%$ \\
\hline $33-37$ & 43 & $17 \%$ & 63 & $23 \%$ \\
\hline $38-42$ & 37 & $15 \%$ & 60 & $22 \%$ \\
\hline $43-47$ & 14 & $6 \%$ & 23 & $9 \%$ \\
\hline+2 & 13 & $5 \%$ & 26 & $10 \%$ \\
\hline $53-57$ & 8 & $3 \%$ & 4 & $1 \%$ \\
\hline $58-62$ & 4 & $2 \%$ & 3 & $1 \%$ \\
\hline $63-67$ & 1 & $0 \%$ & 0 & $0 \%$ \\
\hline $68-72$ & 0 & $0 \%$ & 0 & $0 \%$ \\
\hline $73-77$ & 0 & $0 \%$ & 0 & $0 \%$ \\
\hline $78-82$ & 1 & $0 \%$ & 0 & $0 \%$ \\
\hline Total & 246 & $100 \%$ & 269 & $100 \%$ \\
\hline \multicolumn{5}{|l|}{ No Sexual Partners } \\
\hline 1 & 245 & $100 \%$ & 214 & $79.6 \%$ \\
\hline 2 & 1 & $0 \%$ & 50 & $18.6 \%$ \\
\hline 3 & 0 & $0 \%$ & 5 & $1.9 \%$ \\
\hline Total & 246 & $100 \%$ & 269 & $100 \%$ \\
\hline \multicolumn{5}{|l|}{ Partner HIV status } \\
\hline Positive & 77 & $31 \%$ & 29 & $11 \%$ \\
\hline Negative & 169 & $69 \%$ & 241 & $89 \%$ \\
\hline Total & 246 & $100 \%$ & 270 & $100 \%$ \\
\hline \multicolumn{5}{|l|}{ Marital Status } \\
\hline Unmarried partner & 53 & $22 \%$ & 97 & $36 \%$ \\
\hline Married partner & 193 & $78 \%$ & 173 & $64 \%$ \\
\hline Total & 246 & $100 \%$ & 270 & $100 \%$ \\
\hline
\end{tabular}

Table 3: Index Case Viral load vs Demography of Index Cases and Sexual Partners 


\section{Discussion}

A total of 705 HIV patients enrolled in care at the two centers accessed the HIV partner notification services (PNS) of the hospital during the study period. These clients constituted the index cases who were counselled and brought their 719 sexual partners to the hospitals for HIV testing. About $68 \%$ of the index cases were female and $32 \%$ male while the reverse was the case with the sexual partners who were $67 \%$ male and $33 \%$ females. This shows that virtually all the index cases were able to bring their partners for HIV testing, implying that the PNS was effective and acceptable to the clients and their partners. Thus confirming PNS as an effective strategy in linking up sexual partners to HIV testing services. Up to $71 \%$ of the index cases were in marital relationship with their sexual partners while $29 \%$ were not married to their sexual partners. This figure corroborates the high prevalence of marriage in a culture that prioritize marriage and frown at sexual intercourse outside marriage. While all the index cases were HIV positive, only $27 \%$ of the sexual partners were HIV infected $71 \%$ were HIV negative and $2 \%$ were of unknown HIV status because they did not take the HIV test even after undergoing pre-test counselling. This implies HIV seroconcordance rate of $27 \%$ and discordant rate of $71 \%$. Several African studies, including three trials, have shown partner notification services as an effective strategy for increasing uptake of partner testing and identifying and treating new HIV-cases (10). The HIV prevalence of $27 \%$ among the sexual partners was lower than the prevalence from other studies on PNS such as the Zimbabwean study with HIV positivity yield of $32.6 \%$ among sexual partners (11) but higher than the prevalence found in the Barcelona study (12). But the 2\% partners with unknown HIV status is similar to finding from other study in the same setting (13) A study comparing HIV positivity yield from PNS and other HIV testing strategies shows that PNS significantly yields better than provider-initiated counselling and testing (PITC) and testing at drug stores and private laboratories. The modal age of the sexual partners tested for HIV in this study was between 38 and $42 \mathrm{yrs}$, which account for about $26 \%$ of the partners. Likewise, the most of the concordant and discordant partners are within the same age range. About $48 \%$ of the index cases had suppressed viral load while a slightly more number $(52 \%)$ were virally unsuppressed. This was not significantly different from the $43.1 \%$ prevalence of viral load suppression (VLS) among PLHIV age 15-64 years in Nigeria as documented in NAISS 2018 report. More female are virally suppressed than men( $71 \%$ vs $29 \%)$.Likewise more women had unsuppressed viral load compared to men(64\% vs $36 \%$ ). Most index cases(28\%) with suppressed viral load are within the age range of 28-32yrs while an older age group of $33-$ $37 \mathrm{yrs}$ accounted for most cases $(23 \%)$ with unsuppressed viral load cases .About $100 \%$ of the index with suppressed viral load have one sexual partners while for index cases with unsuppressed viral load $80 \%$ had one sexual partner, $19 \%$ had two sexual partners and $1 \%$ had 3 sexual partners. Therefore, the prevalence of multiple sexual partners was higher with index cases whose viral load was not suppressed. This behaviour could partially explain the unsuppressed viral load in that it is a risk factor for both primary and secondary
HIV (re-infection) infection and same risky behaviour could be a pointer to other risk like non-adherence to antiretroviral therapy, which is primary cause of unsuppressed for PLHIV on treatment. Similarly, incidence of unmarried sexual partners in index cases with unsuppressed viral load was higher at $36 \%$ than with index cases with suppressed viral load (22\%). This is an indication of extra marital sexual relationships among cases with unsuppressed viral load, which is an added risk to their unsuppressed viral load status. However, incidence of HIV infection was higher at $36 \%$ among partners of index cases with suppressed viral load when compared with HIV incidence of $11 \%$ among partners of index with unsuppressed viral load. This is contrary to findings from many other studies which showed unsuppressed viral load as a risk factor to HIV transmission. The presence of other risk factors with overriding effect on viral load may the plausible explanation for this finding.

\section{Conclusion and Recommendations}

All the index cases counselled for PNS accepted and actually brought their partners for HIV testing services at the facilities., showing PNS as a very effective strategy in increasing HIV testing services. Also $98 \%$ of the sexual contacted underwent HIV testing services with their status diagnosed. The HIV positivity yield of $27 \%$ was higher than the yield from routine walk-in clients at the centers. The unsuppressed viral load status of the index cases did not seem to contribute to high HIV positivity yield among the sexual partners.

It is therefore recommended that PNS be made the standard of care at all centers offering HIV testing services.

\section{References}

1. UNAIDS DATA 2020.

2. Nigeria National HIV/AIDS Indicator and Impact Survey (NAIIS) 2018.

3. Revised National HIV and AIDS Strategic Framework 2019-2021, National Agency for the Control of AIDS, Abuja, Nigeria.

4. Kimbrough LW, Fisher HE, Jones KT, Johnson W, Thadiparthi S, Dooley S (2009). Accessing social networks with high rates of undiagnosed HIV infection: The social networks demonstration project. Am J Public Health, 99:1093-1099. 10.2105/AJPH.2008.139329.

5. Wykoff RF, Heath CW, Hollis SL, Leonard ST, Quiller CB, Jones JL, Artzrouni M, Parker RL (1988). Contact tracing to identify human immunodeficiency virus infection in a rural community. JAMA, 259:3563-3566. 10.1001/ jama.1988.03720240025029.

6. Centers for Disease Control and Prevention: Social Networks Testing. A community-based strategy for identifying persons with undiagnosed HIV infection. Interim Guide for HIV Counselling, Testing, and Referral Programs. http://www.cdc.gov/Hiv/resources/guidelines/ snt/index.htm,

7. Woodhouse DE, Rothenberg RB, Potterat JJ, Darrow WW, Muth SQ, Klovdahl AS, Zimmerman HP, Rogers 
HL, Maldonado TS, Muth JB (1994). Mapping a social network of heterosexuals at high risk for HIV infection. AIDS, 8:1331-1336. 10.1097/00002030-19940900000018.

8. Ministry of Health of China, UNAIDS, WHO (2010). The 2009 Estimates for the HIV/AIDS Epidemic in China. Beijing: Ministry of Health, People's Republic of China. http://www.unaids.org.cn/download/2009\%20China\%20 Estimation\%20Report-En.pdf.

9. Lin H, He N, Ding Y, et al (2012). Tracing sexual contacts of HIV-infected individuals in a rural prefecture, Eastern China. BMC Public Health, 12: 533. https://doi. org/10.1186/1471-2458-12-533.

10. Brown LB, Miller WC, Kamanga G, Nyirenda N, Mmodzi P, Pettifor A, Dominik R C, Kaufman JS, Mapanje C, Martinson F, Cohen MS, \& Hoffman IF (1999). HIV partner notification is effective and feasible in sub-Saharan Africa: opportunities for HIV treatment and prevention. Journal of acquired immune deficiency syndromes, 56(5):437-442. https://doi.org/10.1097/qai.0b013e318202bf7d.

11. Garcia de Olalla P, Mollas E, Barbera MJ, Martin S, Arellano E, Gosch M, et al (2015). Effectivenesss of a pilot partner notification program for new HIV cases in Barcelona, Spain. PLos one, 10(4):e0121536 [2].

12. Mahachi N, Muchedzi A, Tafuma T, Mawora P, Kariuki L, Semo B, Batenanya M, Nyagura T, Ncube G, Merrigan M, Chabikuli O, Mpofu M (2019). Sustained high HIV case-finding through Indextesting and partner notification services: experiences from three provinces in Zimbabwe. Journal of the International AIDS Society, 22. doi.10.1002/ jia2.25321.

13. Afe AJ, Fadero T, Oluokun O (2015). HIV Sero-discordant Couples in Southwest Nigeria: Prevalence and Associated Risk Factors. J Aids HIV Infec 1(1):103. Available from: https://www.researchgate.net/publication/275888409 HIV_Sero Discordant_Couples_in_Southwest_Nigeria Prevalence_and_Associated_Risk_Factors [accessed Jan 14 2021].

Copyright: $@ 2021$ Abayomi Joseph Afe. This is an open-access article distributed under the terms of the Creative Commons Attribution License, which permits unrestricted use, distribution, and reproduction in anymedium, provided the original author and source are credited. 